Bundesgesundheitsbl 2015 · 58:1193-1194

DOI 10.1007/s00103-015-2216-4

Online publiziert: 8. August 2015

(c) Springer-Verlag Berlin Heidelberg 2015

CrossMark

Bekanntmachung des Bundesinstituts für Risikobewertung

\title{
Gesundheitliche Beurteilung von Materialien und Gegenständen für den Lebensmittelkontakt im Rahmen des Lebensmittel- und Futtermittelgesetzbuches
}

\section{Mitteilung}

\section{Farbmittel zum Einfärben von Kunststoffen und anderen Polymeren für Bedarfsgegenstände}

Stand vom 01.02.2015

Die Empfehlung, zuletzt geändert nach dem Stand vom 01.01.2010 [Bundesgesundheitsblatt - Gesundheitsforschung Gesundheitsschutz 53 (2010) 86] wird wie folgt neu gefasst:

Als Farbmittel ${ }^{1}$ im Sinne dieser Empfehlung sind die farbgebenden Substanzen einschließlich der möglicherweise als Träger- und Hilfsstoffe vorhandenen Produkte und der technisch unvermeidbaren Verunreinigungen zu verstehen.

Gegen die Verwendung von Farbmitteln zum Einfärben von Kunststoffen und anderen Polymeren ${ }^{2}$ für Bedarfsgegenstände im Sinne von $\$ 2$ Abs. $6 \mathrm{Nr}$. 1 des Lebensmittel- und Futtermittelgesetzbuches bestehen keine Bedenken, sofern die Farbmittel sich für das Einfärben von Kunststoffen eignen und beim bestimmungsgemäßen Gebrauch der Bedarfsgegenstände auch nicht in sichtbaren Anteilen auf die Lebensmittel über-

\footnotetext{
1 Nach DIN 55943 ist „Farbmittel“ der Sammelbegriff für alle farbgebenden Stoffe.

2 Unter "anderen Polymeren" sind in der vorliegenden Empfehlung Elastomere, ferner hochpolymere Stoffe für Beschichtungen und Bindemittel für Lacke zu verstehen.
}

gehen $^{3}$. Darüber hinaus müssen folgende Anforderungen erfüllt sein:

Werden für Kunststoffe im Sinne der Verordnung (EU) Nr. 10/2011 Farbmittel in Form von Farbmittelzubereitungen ${ }^{4}$ (Präparationen) angewendet, so müssen die in den Farbmittelzubereitungen enthaltenen Bindemittel dieser Verordnung entsprechen.

Für alle gemäß Verordnung (EU) Nr. 10/2011 von der Begriffsbestimmung „Kunststoff“ ausgenommenen Materialien zur Herstellung von Lebensmittelbedarfsgegenständen gilt die vorliegende Empfehlung uneingeschränkt.

Werden Farbmittelzubereitungen für andere Materialien als Kunststoffe unter Verwendung von sonstigen Bindemitteln, Anreibemitteln oder anderen notwendigen Hilfsstoffen hergestellt, so dürfen über die in der Verordnung (EU) Nr. 10/2011 aufgeführten Stoffe hinaus die in der nachfolgenden Tabelle aufgeführten Stoffe allein oder in Mischungen miteinander verwendet werden.

\footnotetext{
3 S. 24. Mitteilung zur Untersuchung von Kunststoffen, Bundesgesundheitsblatt 15 (1972) 285.

4 In der Regel werden organische Pigmente in Form von Farbmittelzubereitungen in Mengen von weniger als $1,0 \%$ und anorganische Pigmente in Form von Farbmittelzubereitungen in Mengen bis zu höchstens 5,0\% in den Kunststoff eingearbeitet.
}

Tabelle Stoffe für Farbmittelzubereitungen für andere Materialien als Kunststoffe

\begin{tabular}{|c|c|}
\hline Substanz & $\begin{array}{l}\text { Maximale Ein- } \\
\text { satzmenge }^{\mathrm{a}}\end{array}$ \\
\hline Oleoyl-N-methylglycin & $0,3 \%$ \\
\hline Tri-2-ethylhexylphosphat & \multirow{3}{*}{$\begin{array}{l}\text { Insgesamt } \\
0,2 \%\end{array}$} \\
\hline $\begin{array}{l}\text { Adipinsäurepolyester mit } \\
\text { 1,3-Butandiol und 1,6-He- } \\
\text { xandiol }\end{array}$ & \\
\hline Ölsäuremonoethanolamid & \\
\hline $\begin{array}{l}\text { Kondensationsprodukte } \\
\text { aromatischer Sulfonsäuren } \\
\text { mit Formaldehyd }\end{array}$ & $0,1 \%$ \\
\hline $\begin{array}{l}\text { Kondensationsprodukt } \\
\text { aus Toluolsulfonamid und } \\
\text { Form-aldehyd }\end{array}$ & $0,1 \%$ \\
\hline $\begin{array}{l}\text { aberechnet auf den eingefärbten } \\
\text { Bedarfsgegenstandes. }\end{array}$ & Teil des \\
\hline
\end{tabular}

Die in der Tabelle aufgeführten Stoffe dürfen nur für einen Übergangszeitraum bis zum 01.01.2018 verwendet werden. Es ist vorgesehen, die Regelungen zu den Formulierungshilfsmitteln für Farbmittel für andere Materialien als Kunststoffe auf Richtwerte für ihren Übergang auf Lebensmittel umzustellen. Für die nach dem Übergangszeitraum weiterhin benötigten Stoffe, die nicht in der Verordnung (EU) Nr. 10/2011 aufgeführt sind, ist ein Antrag entsprechend den Vorgaben des „Note for Guidance "der Europäischen Behörde für Lebensmittelsicherheit zu stellen (siehe: http://www.bfr.bund.de/de/bfr_empfehlungen_zu_materialien_fuer_den_lebensmittelkontakt_ehemals_kunststoffempfehlungen_-447.html). 


\section{Reinheitsanforderungen an Farbmittel}

Färberuße müssen den Reinheitsanforderungen an Ruße gemäß Verordnung (EU) Nr. 10/2011 entsprechen ${ }^{5}$.

Darüber hinaus gelten für Farbmittel gemäß dieser Empfehlung folgende weitere Reinheitsanforderungen:

1. Die in 0,07 N Salzsäure löslichen Anteile, ermittelt nach DIN $53770^{6}$, dürfen (bezogen auf das Farbmittel) für nicht überschreiten.

$\begin{array}{ll}\text { Blei } & 0,01 \% \\ \text { Arsen } & 0,01 \% \\ \text { Quecksilber } & 0,005 \% \\ \text { Selen } & 0,01 \% \\ \text { Barium } & 0,01 \% \\ \text { Chrom } & 0,1 \% \\ \text { Cadmium } & 0,01 \% \\ \text { Antimon } & 0,05 \%\end{array}$

2. Bei spezifikationsgemäßer Anwendung darf eine Freisetzung von primären aromatischen Aminen am fertigen Lebensmittelkontaktmaterial mit einer summarischen Nachweisgrenze von $0,01 \mathrm{mg} / \mathrm{kg}$ nicht nachweisbar sein. Für primäre aromatische Amine, die als Kanzerogene der Kategorien $1 \mathrm{~A}$ und $1 \mathrm{~B}$ nach der CLP-Verordnung (EG) Nr. 1272/2008 eingestuft sind, gilt zusätzlich die Anforderung, dass ihre Freisetzung als Einzelsubstanz mit einer Nachweisgrenze von 0,002 mg/kg Lebensmittel bzw. Lebensmittelsimulanz nicht nachweisbar sein darf. Azofarbstoffe, die in primäre aromatische Amine zerfallen können, die als Kanzerogene der Kategorien $1 \mathrm{~A}$ und $1 \mathrm{~B}$ nach der CLP-Verordnung (EG) Nr. 1272/2008 eingestuft sind, dürfen nicht verwendet werden.

Anmerkung. Die zum Färben von Lebensmitteln zugelassenen Farbstoffe können aus technischen Gründen zum Einfärben von Kunststoffen ungeeignet sein. Die zum Einfärben von Kunststoffen verwendeten Farbmittel müssen je nach Art

5 Prüfung der Extinktion des Cyclohexanextrakts entsprechend 23. Mitteilung zur Untersuchung von Kunststoffen, Bundesgesundheitsblatt 15 (1972) 268.

6 S. DIN 53770: „Prüfung von Pigmenten, Bestimmung der salzsäurelöslichen Anteile", Teile 1 bis 7 sowie 13 und 14 . des einzufärbenden Kunststoffes Temperaturen von etwa $150-300^{\circ} \mathrm{C}$ für die Dauer der Verarbeitung des Kunststoffes aushalten. Die Erfahrung hat gezeigt, dass für die Färbung von Kunststoffen geeignete, in diesen unlösliche Pigmente so fest in der Kunststoffmasse eingebettet sind, dass sie beim Kontakt mit Lebensmitteln nicht aus dem Kunststoff herausgelöst werden. Dagegen besteht bei unsachgemäßer Verwendung von löslichen Farbmitteln die Gefahr, dass diese aus dem Kunststoff auf die Lebensmittel übergehen. 